\title{
Aristotelian Diagrams for Semantic and Syntactic Consequence
}

\author{
Lorenz Demey
}

[Accepted for publication in Synthese.]

\begin{abstract}
Several authors have recently studied Aristotelian diagrams for various metatheoretical notions from logic, such as tautology, satisfiability, and the Aristotelian relations themselves. However, all these metalogical Aristotelian diagrams focus on the semantic (model-theoretical) perspective on logical consequence, thus ignoring the complementary, and equally important, syntactic (proof-theoretical) perspective. In this paper, I propose an explanation for this discrepancy, by arguing that the metalogical square of opposition for semantic consequence exhibits a natural analogy to the wellknown square of opposition for the categorical statements from syllogistics, but that this analogy breaks down once we move from semantic to syntactic consequence. I then show that despite this difficulty, one can indeed construct metalogical Aristotelian diagrams from a syntactic perspective, which have their own, equally elegant characterization in terms of the categorical statements. Finally, I construct several metalogical Aristotelian diagrams that incorporate both semantic and syntactic consequence (and their interaction), and study how they are influenced by the underlying logical system's soundness and/or completeness. All of this provides further support for the methodological/heuristic perspective on Aristotelian diagrams, which holds that the main use of these diagrams lies in facilitating analogies and comparisons between prima facie unrelated domains of investigation.
\end{abstract}




\section{Introduction}

Aristotelian diagrams are visual representations of a set of propositions, concepts or expressions, and the logical relations holding between them. Without a doubt, the oldest and most widely used example is the so-called 'square of opposition', but there also exist many larger, more complex Aristotelian diagrams. Throughout the history of philosophy and logic, distinguished authors such as William of Ockham, Gottfried Leibniz and Gottlob Frege have made use of such diagrams to illustrate and explain their theorizing (Kienzler 2013, Lenzen 2016, Parsons 2017). In $20^{\text {th }}$ century analytic philosophy, Aristotelian diagrams have been used in fields as diverse as philosophy of action (Kenny 1963), ethics (Chisholm 1963), philosophy of language (Hare 1967), philosophy of law (Hart 1982), epistemology (Sosa 1964) and philosophy of religion (Hess 2017). They are also widely used to study families of logics such as modal logic (Rini and Cresswell 2012), relevant logic (Beall et al. 2006), deontic logic (Barcan Marcus 1966) and probabilistic logic (Pfeifer and Sanfilippo 2017). Furthermore, because of the ubiquity of the logical relations that they visualize, Aristotelian diagrams are nowadays also frequently used beyond philosophy and logic, in disciplines such as computer science (Ciucci et al. 2016), law (Vranes 2006) and linguistics (Ziegeler 2017).

The received view holds that Aristotelian diagrams primarily function as pedagogical devices for introducing novice students to logic. However, this view has become untenable, because today, most Aristotelian diagrams are no longer found in introductory logic textbooks, but rather in research papers and monographs (cf. the examples cited above). An alternative explanation for the widespread use of Aristotelian diagrams is based on their methodological importance. For example, Yao (2013), Ciucci et al. (2014, 2016) and Dubois et al. (2015) have pointed out the heuristic usefulness of Aristotelian diagrams in the theoretical foundations of artificial intelligence, emphasizing their role in drawing comparisons across individual knowledge representation formalisms and in discovering new notions (by transferring them across formalisms). Demey (2017b, 2018a, 2018c) and Demey and Smessaert (2018) generalize these remarks to the applicability of Aristotelian diagrams in (drawing comparisons between) other areas, such as Russell's theory of definite descriptions and public announcement logic.

Most of the Aristotelian diagrams that have appeared in the logical literature thus far are situated at the object-logical level: the propositions appearing in these diagrams come from the object language of the logical system for which the diagram is drawn. However, in recent years there 
has been a surge of interest in Aristotelian diagrams at the metalogical level, which contain statements or notions belonging to the metatheory of a given logical system. For example, Béziau (2012, 2013) and Diaconescu (2015) have studied Aristotelian diagrams for metalogical notions such as tautology, contradiction, satisfiability, etc. Furthermore, Béziau (2012, 2016), Seuren (2014) and Demey and Smessaert (2016) have constructed diagrams that represent the Aristotelian relations holding between the Aristotelian relations themselves; Demey and Smessaert (2014) and Demey (2018b) have used these diagrams to study the specific language used in metalogic from the perspective of (neo-)Gricean pragmatics. Finally, it bears emphasizing that these metalogical applications of Aristotelian diagrams have been motivated by pedagogical as well as methodological considerations. For example, Demey (2017a) has argued that such diagrams can be fruitfully used to teach metalogic to certain types of students, while Demey (2018b) has used them to draw a linguistic comparison between object- and metalogical terminology.

Despite this recent surge of interest, all metalogical Aristotelian diagrams studied thus far focus exclusively on the semantic (model-theoretical) perspective on logic and logical consequence (cf. Demey 2017a, Footnote 5). So far, no one seems to have studied metalogical Aristotelian diagrams for the complementary, and equally important, syntactic (proof-theoretical) perspective on logic and logical consequence. ${ }^{1}$ This omission is particularly unfortunate for the aforementioned methodological/heuristic perspective on Aristotelian diagrams, which emphasizes the role of these diagrams in connecting and comparing different frameworks. After all, in light of the well-known soundness and completeness theorems, which establish a strong connection between syntactic and semantic logical consequence, one should be able to construct Aristotelian diagrams for syntactic as well as for semantic logical consequence, and also for their interaction. The current asymmetry (there exist many Aristotelian diagrams for semantic consequence, but none for syntactic consequence) thus seems to present a counterexample to the methodological/heuristic account of the widespread use Aristotelian diagrams.

My main aim in this paper is to address this important lacuna in the literature on (metalogical) Aristotelian diagrams. This naturally leads to a sequence of interrelated subgoals. First of all, I

\footnotetext{
${ }^{1}$ Diaconescu (2015) studies several metalogical Aristotelian diagrams for abstract (Tarski-style) consequence relations, which transcend the distinction between syntax and semantics. However, he does not deal with any Aristotelian diagrams for syntactic consequence in particular.
} 
will propose an explanation as to why all research in this area has thus far focused exclusively on the semantic perspective. In particular, I will argue that the metalogical square of opposition for semantic consequence exhibits a natural analogy to the oldest and most well-known square of opposition, viz. that for the categorical statements from Aristotelian syllogistics. ${ }^{2}$ By contrast, this analogy breaks down quite spectacularly once we move from semantic to syntactic consequence. The second subgoal is to show that despite this prima facie difficulty, one can indeed construct metalogical Aristotelian diagrams from a syntactic perspective. Furthermore, these new diagrams have their own, equally theoretically elegant characterization in terms of the categorical statements. We thus obtain Aristotelian diagrams for semantic consequence on the one hand, and for syntactic consequence on the other (both of which exhibit a strong analogy with the categorical statements from syllogistics). The paper's third and final subgoal is therefore to construct metalogical Aristotelian diagrams that incorporate both semantic and syntactic consequence (and their interaction), and to study how these diagrams are influenced by the underlying logical system's soundness and/or completeness.

The paper is organized as follows. In Section 2, I briefly rehearse the most important metalogical Aristotelian diagram for semantic consequence that has been studied in the literature, and argue that it can naturally be characterized in terms of the categorical statements from syllogistics. Next, in Section 3, I show that one can construct an analogous metalogical Aristotelian diagram for syntactic consequence. I also consider the most straightforward way of characterizing this new diagram in terms of the categorical statements, and argue that this fails for various reasons. In Section 4, I then propose an alternative way of understanding the new diagram for syntactic consequence in terms of the categorical statements, and show that this new interpretation is indeed successful. I also discuss why this alternative characterization of the diagram for syntactic consequence is arguably the closest possible analogue of the characterization of the diagram for semantic consequence. Building on these results, in Section 5, I construct several metalogical Aristotelian diagrams that incorporate both semantic and syntactic consequence (and any potential interaction between them). I also study how the soundness and/or completeness of the underlying logical system influence the metalogical

\footnotetext{
${ }^{2}$ Note that this argumentation for an analogy (between the metalogical notion of semantic consequence and the categorical statements from syllogistics) is itself perfectly in line with the methodological/heuristic perspective on Aristotelian diagrams.
} 
properties of these diagrams. Finally, in Section 6, I briefly summarize the paper, and offer some suggestions for future work.

\section{The Metalogical Square of Opposition for Semantic Consequence}

In this section I will discuss the metalogical Aristotelian diagram for semantic consequence that has been used most widely in the literature. First, however, I will briefly make some basic remarks about the Aristotelian relations and Aristotelian diagrams in general.

The Aristotelian relations can be defined on various levels of generality and abstractness. The most general definition is formulated in terms of arbitrary Boolean algebras; this definition enables us to capture very precisely the similarities and differences between two types of Aristotelian relations, viz. those between object-logical notions and those between metalogical notions (Demey and Smessaert 2016, Demey 2018b). However, for our current purposes it will suffice to define the Aristotelian relations in the usual, more informal way. Two statements are said to be:

- contradictory iff they cannot be true together, and

they cannot be false together;

- contrary iff they cannot be true together, but they can be false together;

- subcontrary iff they cannot be false together, but they can be true together;

- in subalternation iff the first statement entails the second one, but the second statement does not entail the first one.

An Aristotelian diagram visually represents a finite number of statements, together with the Aristotelian relations holding between those statements. The Aristotelian relations are visualized according to the code shown in Figure 1. The oldest example of an Aristotelian diagram is the square of opposition for the four categorical statements from syllogistics, which is also shown in Figure 1. In particular, the A-statement 'all S are P' and the O-statement 'some $\mathrm{S}$ are not $\mathrm{P}$ ' are contradictory; the A-statement 'all $\mathrm{S}$ are $\mathrm{P}$ ' and the E-statement 'no $\mathrm{S}$ are $\mathrm{P}$ ' 
are contrary, and so on. ${ }^{3}$ Note that we are implicitly assuming the principle of existential import - i.e., that there exists at least one $\mathrm{S} .{ }^{4}$ Without this assumption, all Aristotelian relations, except for the two contradiction relations (A/O and E/I), would fail to hold, and hence, the classical square of opposition for the categorical statements would turn into a 'degenerate square' (or ' $\mathrm{X}$ of opposition'; Béziau and Payette 2012, pp. 11-12), in which only the two contradiction relations are left. This constitutes a prime example of a fact that is well-known in logical geometry - i.e. the systematic study of Aristotelian diagrams -, viz. the fact that these diagrams are highly sensitive with respect to certain background assumptions (Demey 2015, Demey and Smessaert 2018).

\section{$<$ INCLUDE FIGURE 1 HERE $>$}

$<$ CAPTION: Figure 1: (left) visual code for representing the Aristotelian relations; (right) square of opposition for the categorical statements from syllogistics, under the assumption of existential import.>

We now turn to the metalogical square of opposition for semantic consequence. To this end, we consider a logical system $S$, with an object language $\mathcal{L}_{S}$, which is supposed to have a classical negation $(\neg)$ and a model-theoretic semantics. This involves a collection $\mathcal{C}_{\mathrm{S}}$ of models ${ }^{5}$ and a binary relation $\vDash$ between the models in $\mathcal{C}_{\mathrm{S}}$ and the formulas in $\mathcal{L}_{\mathrm{S}}$, where $M \vDash \varphi$ means that the formula $\varphi$ is true in the model $M$. We write $M \vDash \varphi$ to abbreviate that it is not the case that $M \vDash \varphi$. The classicality of negation means that $M \vDash \neg \varphi$ iff $M \vDash \varphi$, for every $M \in \mathcal{C}_{\mathrm{S}}$. Furthermore, if $\Gamma$ is a set of $\mathcal{L}_{\mathrm{S}}$-formulas, we write $M \vDash \Gamma$ to abbreviate that $M \vDash \gamma$ for every $\gamma \in \Gamma$. We can now define the notions of semantic logical consequence and satisfiability in the logical system $\mathrm{S}$. Given any set $\Gamma$ of $\mathcal{L}_{\mathrm{S}}$-formulas, and $\mathcal{L}_{\mathrm{S}}$-formula $\varphi$, we say that:

- $\varphi$ is a semantic consequence of $\Gamma$ (notation: $\Gamma \vDash \varphi$ ) iff for all models $M \in \mathcal{C}_{\mathrm{S}}$, it holds that if $M \vDash \Gamma$, then $M \vDash \varphi$;

- $\quad \Gamma$ is satisfiable iff there exists at least one model $M \in \mathcal{C}_{\mathrm{S}}$ such that $M \vDash \Gamma$.

\footnotetext{
${ }^{3}$ Throughout this paper I will make use of the well-known mnemonic vowels (A, I, E, O) for the categorical statements. These are the first two vowels from each of the Latin verb forms 'affirmo' and 'nego'.

${ }^{4}$ The precise interpretation of the principle of existential import in syllogistics has been a matter of substantial scholarly debate (Parsons 2017), but this need not concern us here.

${ }^{5}$ In particular, these models can be first-order models, relational structures, etc. These details do not matter here.
} 
Finally, we write $\Gamma \not \models \varphi$ to abbreviate that it is not the case that $\Gamma \vDash \varphi$.

Béziau (2012, 2013) and Diaconescu (2015) show that if $\Gamma$ is satisfiable, then one can construct a metalogical square of opposition for semantic consequence, as shown in Figure 2. For example, $\Gamma \vDash \varphi$ is contrary to $\Gamma \vDash \neg \varphi$, since these statements cannot be true together (if simultaneously $\Gamma \vDash \varphi$ and $\Gamma \vDash \neg \varphi$, then $\Gamma$ would not be satisfiable), but they can be false together. Note that in order to establish any of the Aristotelian relations in this square of opposition (except for the two contradiction relations), we need to rely on the assumption that $\Gamma$ is satisfiable. Without this assumption, the metalogical square for semantic consequence would thus not be a classical square of opposition, but rather a degenerate square. This can be seen as a metalogical manifestation of the sensitivity of Aristotelian diagrams with respect to certain background assumptions (cf. supra).

\section{$<$ INCLUDE FIGURE 2 HERE $>$}

$<$ CAPTION: Figure 2: metalogical square of opposition for semantic consequence, under the assumption that $\Gamma$ is satisfiable. $>$

It has recently been pointed out that the definitions of the semantic consequence statements appearing in Figure 2 can all be interpreted as categorical statements (Demey 2017a). In particular, recall that the definition of $\Gamma \vDash \varphi$ is:

for all models $M \in \mathcal{C}_{\mathrm{S}}$, it holds that if $M \vDash \Gamma$, then $M \vDash \varphi$.

This definition can be interpreted as a categorical A-statement: it is of the form 'all S are P', with the subject term S standing for 'being a model $M \in \mathcal{C}_{\mathrm{S}}$ such that $M \vDash \Gamma$ ', and the predicate term $\mathrm{P}$ standing for 'being a model $M \in \mathcal{C}_{\mathrm{S}}$ such that $M \vDash \varphi$ '. Furthermore, consider the definition of $\Gamma \vDash \neg \varphi$ :

for all models $M \in \mathcal{C}_{\mathrm{S}}$, it holds that if $M \vDash \Gamma$, then $M \vDash \neg \varphi$.

Since $M \vDash \neg \varphi$ iff $M \vDash \varphi$ (for every $M \in \mathcal{C}_{\mathrm{S}}$ ), this can be reformulated as follows: 
for all models $M \in \mathcal{C}_{\mathrm{S}}$, it holds that if $M \vDash \Gamma$, then $M \vDash \varphi$.

The definition of $\Gamma \vDash \neg \varphi$ can thus be interpreted as a categorical E-statement: after a minor reformulation, it is of the form 'all $\mathrm{S}$ are not $\mathrm{P}$ ' (i.e.: 'no $\mathrm{S}$ are $\mathrm{P}$ '), with $\mathrm{S}$ and $\mathrm{P}$ exactly as above. Similarly, the definition of $\Gamma \not \models$ is:

there exists at least one model $M \in \mathcal{C}_{\mathrm{S}}$ such that $M \vDash \Gamma$ and $M \vDash \varphi$.

This definition can be interpreted as a categorical O-statement: it is of the form 'some $\mathrm{S}$ are not P', with S and P as above. Finally, consider the definition of $\Gamma \not \neg \varphi$ :

there exists at least one model $M \in \mathcal{C}_{\mathrm{S}}$ such that $M \vDash \Gamma$ and $M \not \neg \varphi$.

Since $M \vDash \neg \varphi$ iff $M \vDash \varphi$ (for every $M \in \mathcal{C}_{\mathrm{S}}$ ), this can be reformulated as follows:

there exists at least one model $M \in \mathcal{C}_{\mathrm{S}}$ such that $M \vDash \Gamma$ and $M \vDash \varphi$.

The definition of $\Gamma \not \neq \neg \varphi$ can thus be interpreted as a categorical I-statement: after a minor reformulation, it is of the form 'some $\mathrm{S}$ are $\mathrm{P}$ ', with $\mathrm{S}$ and $\mathrm{P}$ again as above.

The definitions of the four statements regarding semantic consequence can thus be characterized in terms of the four categorical statements. The subject and predicate term in these categorical statements involve the most essential semantic notion, viz. truth (in a model). This characterization establishes a direct link between the squares of opposition in Figures 1 and 2. For example, $\Gamma \vDash \varphi$ and $\Gamma \vDash \neg \varphi$ are contrary to each other in the square of opposition for semantic consequence in Figure 2, and the definitions of these two statements can be interpreted as A- and E-statements, respectively, which are contrary to each other in the square of opposition for the categorical statements in Figure 1. Similarly, $\Gamma \not \varphi$ and $\Gamma \not \neg \varphi$ are subcontrary to each other in the square in Figure 2, and the definitions of these two statements can be interpreted as O- and I-statements, respectively, which are subcontrary to each other in the square in Figure 1. Furthermore, this characterization also shows that the assumption that $\Gamma$ is satisfiable (i.e. there exists at least one model $M \in \mathcal{C}_{\mathrm{S}}$ such that $M \vDash \Gamma$ ) is analogous to the assumption of existential import in syllogistics (i.e. there exists at least one S). Based on the 
definitions of its notions, the metalogical square of opposition for semantic consequence thus turns out to be perfectly analogous to the most widely known square of opposition in the literature, viz. that for the categorical statements from syllogistics.

It should be pointed out that this characterization of semantic consequence in terms of categorical statements crucially relies on the fact that we can shift negation between the objectand the metalogical level $(M \vDash \neg \varphi$ iff $M \vDash \varphi)$. This is essential for reformulating the definitions of $\Gamma \vDash \neg \varphi$ and $\Gamma \not \neg \varphi$ from statements about models that make the negated formula $\neg \varphi$ true or not into statements about models that make the formula $\varphi$ itself true or not. The latter can then be interpreted as E- and I-statements, on a par with the A- and O-statements that correspond to $\Gamma \vDash \varphi$ and $\Gamma \not \models$, respectively.

Once the analogy between semantic consequence and the categorical statements is in place, it can fruitfully be used to understand a certain phenomenon on one side of the analogy in terms of the corresponding phenomenon on the other side of the analogy. For example, in Demey (2017a) this analogy is used to explain that the entailment from $\Gamma \vDash \varphi$ to $\Gamma \not \neg \varphi$ crucially depends on the assumption that $\Gamma$ be satisfiable, by drawing a comparison to the entailment from the A- to the I-statement and its dependence on the assumption of existential import. Such an explanation can be very helpful, for example for philosophers who are thoroughly familiar with syllogistics, but not with metalogic. These observations are perfectly in line with the methodological/heuristic perspective on Aristotelian diagrams (also recall Footnote 2).

\section{The Metalogical Square of Opposition for Syntactic Consequence}

I will now introduce a metalogical square of opposition for syntactic consequence. To this end, we assume that our logical system $\mathrm{S}$ also has a natural deduction proof system $\mathcal{D}_{\mathrm{S}}$, which allows us to construct derivations that lead from (sets of) $\mathcal{L}_{\mathrm{S}}$-formulas to other $\mathcal{L}_{\mathrm{S}}$-formulas. ${ }^{6} \mathrm{We}$ can now define the notions of syntactic logical consequence and consistency in S. Given any set $\Gamma$ of $\mathcal{L}_{\mathrm{S}}$-formulas, and $\mathcal{L}_{\mathrm{S}}$-formula $\varphi$, we say that:

\footnotetext{
${ }^{6}$ In particular, the system can be a Gentzen-style or a Fitch-style natural deduction system. Again, these details do not matter here. (Also see Footnote 5.)
} 
- $\varphi$ is a syntactic consequence of $\Gamma$ (notation: $\Gamma \vdash \varphi$ ) iff

there exists at least one derivation in $\mathcal{D}_{\mathrm{S}}$ that starts from formulas in $\Gamma$ and ends in $\varphi$;

- $\quad \Gamma$ is consistent iff there does not exist a formula $\gamma \in \mathcal{L}_{\mathrm{S}}$ such that $\Gamma \vdash \gamma$ and $\Gamma \vdash \neg \gamma$;

- $\quad \Gamma$ is consistent with $\varphi$ iff the set $\Gamma \cup\{\varphi\}$ is consistent;

- $\quad \Gamma$ is a maximal consistent set (MCS) iff $\Gamma$ is consistent and for all formulas $\gamma \in \mathcal{L}_{\mathrm{S}}$ : if $\gamma \notin \Gamma$ then $\Gamma \cup\{\gamma\}$ is not consistent.

We will write $\Gamma \Vdash \varphi$ to abbreviate that it is not the case that $\Gamma \vdash \varphi$. Furthermore, we will assume that the proof system $\mathcal{D}_{\mathrm{S}}$ has the property that if $\Gamma \cup\{\varphi\}$ is not consistent (i.e., if $\Gamma$ is not consistent with $\varphi$ ), then $\Gamma \vdash \neg \varphi$; this will play an important role in (the proofs of) Theorems 1 and 2 in Section 4. Classical natural deduction proof systems indeed have this property (as well as its converse).

Under ideal circumstances, semantic and syntactic consequence should be closely related to each other - after all, both are meant to capture a single, informal notion of logical consequence. Given the square of opposition for semantic consequence from Section 2, one would therefore expect that there exists an analogous square of opposition for syntactic consequence, as shown in Figure 3. It is easy to prove that if $\Gamma$ is consistent, then all the Aristotelian relations in Figure 3 indeed hold, i.e. we indeed obtain a square of opposition for syntactic consequence. For example, $\Gamma \vdash \varphi$ is contrary to $\Gamma \vdash \neg \varphi$, since these statements cannot be true together (if simultaneously $\Gamma \vdash \varphi$ and $\Gamma \vdash \neg \varphi$, then $\Gamma$ would not be consistent), but they can be false together. Note that in order to establish any of the Aristotelian relations in this square of opposition (except for the two contradiction relations), we need to rely on the assumption that $\Gamma$ is consistent. Without this assumption, the metalogical square for syntactic consequence would not be a classical square of opposition, but rather a degenerate square. This can be seen as yet another metalogical manifestation of the sensitivity of Aristotelian diagrams with respect to certain background assumptions (cf. supra).

$<$ INCLUDE FIGURE 3 HERE $>$

$<$ CAPTION: Figure 3: metalogical square of opposition for syntactic consequence, under the assumption that $\Gamma$ is consistent.> 
Let us now have a closer look at the definitions of the syntactic consequence statements that appear in Figure 3. First of all, recall that the definition of $\Gamma \vdash \varphi$ is:

there exists at least one derivation in $\mathcal{D}_{\mathrm{S}}$ that starts from formulas in $\Gamma$ and ends in $\varphi$.

Completely analogously, the definition of $\Gamma \vdash \neg \varphi$ is:

there exists at least one derivation in $\mathcal{D}_{\mathrm{S}}$ that starts from formulas in $\Gamma$ and ends in $\neg \varphi$.

Furthermore, here are three equivalent formulations of the definition of $\Gamma \Vdash \varphi$ :

there does not exist a derivation in $\mathcal{D}_{\mathrm{S}}$ that starts from formulas in $\Gamma$ and ends in $\varphi$, no derivation in $\mathcal{D}_{\mathrm{S}}$ that starts from formulas in $\Gamma$, ends in $\varphi$, all derivations in $\mathcal{D}_{\mathrm{S}}$ that start from formulas in $\Gamma$, do not end in $\varphi$.

Finally, consider three equivalent formulations of the definition of $\Gamma \Vdash \neg \varphi$ :

there does not exist a derivation in $\mathcal{D}_{\mathrm{S}}$ that starts from formulas in $\Gamma$ and ends in $\neg \varphi$, no derivation in $\mathcal{D}_{\mathrm{S}}$ that starts from formulas in $\Gamma$, ends in $\neg \varphi$, all derivations in $\mathcal{D}_{\mathrm{S}}$ that start from formulas in $\Gamma$, do not end in $\neg \varphi$.

If we try to characterize these definitions of the four syntactic consequence statements in terms of the four categorical statements from syllogistics, we immediately encounter several problems. First of all, there is a 'quantificational mismatch'. For example, since $\Gamma \vdash \varphi$ and $\Gamma \vdash$ $\neg \varphi$ are contraries in the square of opposition for syntactic consequence in Figure 3, we should expect their definitions to correspond to the universally quantified A- and E-statements, which are contraries in the square of opposition for the categorical statements in Figure 1. However, we have just seen that the definitions of $\Gamma \vdash \varphi$ and $\Gamma \vdash \neg \varphi$ involve an existential, rather than a universal quantification ('there exists at least one derivation...'). Similarly, since $\Gamma \Vdash \varphi$ and $\Gamma \nvdash \neg \varphi$ are subcontraries in the square in Figure 3, we should expect their definitions to correspond to the existentially quantified O- and I-statements, which are subcontraries in the 
square in Figure 1. However, we have just seen that the definitions of $\Gamma \Vdash \varphi$ and $\Gamma \Vdash \neg \varphi$ involve a universal, rather than an existential quantification ('all derivations...').

This quantificational mismatch can also be discerned in the assumption that is needed to prove that the square is indeed a classical square of opposition (rather than a degenerate square). In the case of the categorical statements in Figure 1, this is the assumption of existential import, which, as the name already suggests, is an existential claim ('there exists at least one S'). In the case of the semantic consequence statements in Figure 2, the assumption concerns the satisfiability of $\Gamma$, which is also an existential claim ('there exists at least one model $M$...'). By contrast, in the case of the syntactic consequence statements in Figure 3, the assumption concerns the consistency of $\Gamma$, which is a universal, rather than an existential claim ('there does not exist a formula $\gamma \ldots$..').

The final, and perhaps most serious problem, concerns the behavior of negation. Regardless of their quantificational status, the definitions of all four syntactic consequence statements share the same subject term, viz. 'being a derivation in $\mathcal{D}_{\mathrm{S}}$ that starts from formulas in $\Gamma$ '. However, they do not share the same predicate term: the predicate term in the definitions of $\Gamma \vdash \varphi$ and $\Gamma \nvdash \varphi$ is 'being a derivation in $\mathcal{D}_{\mathrm{S}}$ that ends in $\varphi$ ', whereas the predicate term in the definitions of $\Gamma \vdash \neg \varphi$ and $\Gamma \Vdash \neg \varphi$ is 'being a derivation in $\mathcal{D}_{\mathrm{S}}$ that ends in $\neg \varphi$ '. The latter predicate term cannot be reduced to the former, since ending in $\neg \varphi$ is strictly stronger than not ending in $\varphi$. (If a derivation ends in $\neg \varphi$, it trivially does not end in $\varphi$; however, the converse does not hold: a derivation might end in some third formula $\psi$, in which case it does not in $\varphi$, and not in $\neg \varphi$ either.) This should be contrasted with the definitions of the semantic consequence statements in Section 2, which do share a single (subject term and) predicate term, since $M \vDash \neg \varphi$ is indeed equivalent to $M \not \varphi$.

\footnotetext{
${ }^{7}$ The problematic nature of this quantifier mismatch should not be exaggerated. Ultimately, $\Gamma \vdash \varphi$ and $\Gamma \vdash \neg \varphi$ are effectively contrary to each other, regardless of whether their definitions can be characterized as universal (Aand E-) statements. In the literature there exist other, object-logical examples of squares of opposition that exhibit a similar quantifier mismatch. For example, in the square of opposition for public announcement logic, the formulas $<! p>q$ and $<! p>\neg q$ are contrary to each other, although the semantics of these formulas involves an existential, rather than a universal quantification (over public announcements of $p$ ) (Demey 2012, 2017b).
} 
The overall argument that has been developed in this section and the previous one, can now be summarized as follows:

1. Both semantic and syntactic consequence give rise to a metalogical square of opposition (under analogous metalogical conditions, viz. the satisfiability and consistency of $\Gamma)$.

2. The definitions of the four semantic consequence statements can straightforwardly be interpreted in terms of the four categorical statements from syllogistics. Hence, the square of opposition for semantic consequence in Figure 2 (and its assumption of the satisfiability of $\Gamma$ ) exhibits a strong analogy to the square of opposition for the categorical statements in Figure 1 (and its assumption of existential import).

3. By contrast, the definitions of the four syntactic consequence statements cannot be interpreted in terms of the four categorical statements in the same straightforward fashion. Hence, the square of opposition for syntactic consequence in Figure 3 (and its assumption of the consistency of $\Gamma$ ) does not exhibit a strong analogy to the square of opposition for the categorical statements in Figure 1 (and its assumption of existential import).

Taking into consideration that the square of opposition for the categorical statements from syllogistics is by far the oldest and most well-known Aristotelian diagram, and thus serves as a kind of 'golden standard', I would like to suggest that this discrepancy between the semantic consequence square and the syntactic consequence square might be precisely the reason why the former has been studied quite frequently in the recent literature on metalogical Aristotelian diagrams, whereas the latter has not been studied at all thus far.

Finally, it bears emphasizing that this discrepancy between the semantic consequence square and the syntactic consequence square is not entirely undesirable. Consider, for example, the quantificational aspects of the discrepancy: the definition of $\Gamma \vDash \varphi$ is a universal claim, while the definition of $\Gamma \vdash \varphi$ is an existential claim. As anyone who has ever taught metalogic will know, this quantificational mismatch can also be highly beneficial. After all, proving that $\Gamma \vdash$ $\varphi$ requires constructing just a single derivation in $\mathcal{D}_{\mathrm{S}}$, and this will often be easier than proving that $\Gamma \vDash \varphi$, which requires considering all models in $\mathcal{C}_{S}$. Vice versa, proving that $\Gamma \vDash \varphi$ requires constructing just a single (counter)model, and this will often be easier than proving 
that $\Gamma \Vdash \varphi$, which requires considering all possible derivations. From this perspective, the quantificational mismatch between semantic and syntactic consequence is thus an advantage, rather than a disadvantage. Nevertheless, for our current purposes - viz. studying the Aristotelian diagrams that semantic and syntactic consequence give rise to - it remains highly desirable to obtain a sound characterization of both semantic and syntactic consequence in terms of the categorical statements.

\section{An Alternative Characterization of the Metalogical Square for Syntactic Consequence}

In the previous section I have argued that although the syntactic consequence statements constitute a metalogical square of opposition, their definitions cannot straightforwardly be understood in terms of the categorical statements. In this section I will propose an alternative characterization of the syntactic consequence statements, and show that it does enable a direct analogy to the categorical statements. ${ }^{8}$ We therefore consider the following:

Theorem 1. Given any set $\Gamma$ of $\mathcal{L}_{\mathrm{S}}$-formulas, and $\mathcal{L}_{\mathrm{S}}$-formula $\varphi$, it holds that:
1) $\Gamma \vdash \varphi$
iff
all MCS $\Delta \supseteq \Gamma \operatorname{contain} \varphi$;
2) $\Gamma \vdash \neg \varphi$
iff
no $\operatorname{MCS} \Delta \supseteq \Gamma \operatorname{contain} \varphi$;
3) $\Gamma \nvdash \varphi$
iff
some MCS $\Delta \supseteq \Gamma$ do not contain $\varphi$;
4) $\Gamma \Vdash \neg \varphi$
iff
some $\operatorname{MCS} \Delta \supseteq \Gamma \operatorname{contain} \varphi$;
5) $\Gamma$ is consistent $\quad$ iff
there exists at least one MCS $\Delta \supseteq \Gamma$.

Proof. Item 1, left to right. Consider an arbitrary MCS $\Delta \supseteq \Gamma$ and suppose, toward a contradiction, that $\Delta$ does not contain $\varphi$. By the maximality of $\Delta$, it follows that $\Delta \cup\{\varphi\}$ is not consistent, and hence $\Delta \vdash \neg \varphi$. Furthermore, since $\Gamma \vdash \varphi$ and $\Gamma \subseteq \Delta$, we also have $\Delta \vdash \varphi$. This violates the consistency of $\Delta$.

Item 1, right to left is proved by contraposition, so we assume that $\Gamma \Vdash \varphi$, and show that there exists an MCS $\Delta \supseteq \Gamma$ that does not contain $\varphi$. Consider the set $\Gamma \cup\{\neg \varphi\}$. Since $\Gamma \Vdash \varphi$, this set is consistent, so by Lindenbaum's lemma there exists an MCS $\Delta \supseteq \Gamma \cup\{\neg \varphi\}$. It now trivially

\footnotetext{
${ }^{8}$ Thanks to $* * *$
} 
holds that $\Delta \supseteq \Gamma$; furthermore, since $\neg \varphi \in \Delta$ and $\Delta$ is consistent, it follows that $\Delta$ does not contain $\varphi$.

Item 2, left to right. Consider an arbitrary $\operatorname{MCS} \Delta \supseteq \Gamma$ and suppose, toward a contradiction, that $\Delta$ contains $\varphi$. It immediately follows that $\Delta \vdash \varphi$. Furthermore, since $\Gamma \vdash \neg \varphi$ and $\Gamma \subseteq \Delta$, we also have $\Delta \vdash \neg \varphi$. This violates the consistency of $\Delta$.

Item 2 , right to left is again proved by contraposition, so we assume that $\Gamma \Vdash \neg \varphi$, and show that there exists an MCS $\Delta \supseteq \Gamma$ that contains $\varphi$. Consider the set $\Gamma \cup\{\varphi\}$. Since $\Gamma \nvdash \neg \varphi$, this set is consistent, so by Lindenbaum's lemma there exists an MCS $\Delta \supseteq \Gamma \cup\{\varphi\}$. It now trivially holds that $\Delta \supseteq \Gamma$ and that $\varphi \in \Delta$.

Items 3 and 4 follow from items 1 and 2, respectively (after all, if two statements A and B are equivalent to each other, then their negations $\neg \mathrm{A}$ and $\neg \mathrm{B}$ are also equivalent to each other).

Item 5, left to right is merely an expression of Lindenbaum's lemma.

Item 5, right to left is proved by contraposition, so we assume that $\Gamma$ is not consistent, and show that there does not exist an MCS $\Delta \supseteq \Gamma$. Since $\Gamma$ is not consistent, there exists a formula $\gamma$ such that $\Gamma \vdash \gamma$ and $\Gamma \vdash \neg \gamma$; hence, for every $\Delta \supseteq \Gamma$ it follows that also $\Delta \vdash \gamma$ and $\Delta \vdash \neg \gamma$, so $\Delta$ is not consistent, and thus a fortiori not an MCS.

The first four items of Theorem 1 provide characterizations of the syntactic consequence statements that correspond to the categorical statements, which all have the same subject term, viz. 'being an MCS $\Delta \subseteq \mathcal{L}_{\mathrm{S}}$ such that $\Gamma \subseteq \Delta$ ', as well as the same predicate term, viz. 'being an $\operatorname{MCS} \Delta \subseteq \mathcal{L}_{\mathrm{S}}$ such that $\varphi \in \Delta^{\prime}$.

This correspondence does not suffer from a quantifier mismatch or problems regarding negation (cf. Section 3). For example, $\Gamma \vdash \varphi$ and $\Gamma \vdash \neg \varphi$ are contraries in the square of opposition for syntactic consequence in Figure 3, and the first two items of Theorem 1 show that these statements correspond to the universally quantified A- and E-statements, respectively, which are contraries in the square of opposition for the categorical statements in Figure 1. Similarly, 
$\Gamma \nvdash \varphi$ and $\Gamma \nvdash \neg \varphi$ are subcontraries in the square in Figure 3, and items 3 and 4 of the theorem show that these statements correspond to the existentially quantified $\mathrm{O}$ - and I-statements, respectively, which are subcontraries in the square in Figure 1. Furthermore, consider the assumption that is needed to prove that the square is indeed a classical square of opposition (rather than a degenerate square). In the case of the syntactic consequence statements, this is the assumption that $\Gamma$ is consistent, and the theorem's fifth item provides a characterization of this assumption as an existential claim, which is completely analogous to the existential import assumption in the case of the categorical statements. Finally, note that there is no problem with negation, as all four characterizations share the same predicate term, viz. 'being an MCS $\Delta \subseteq$ $\mathcal{L}_{\mathrm{S}}$ such that $\varphi \in \Delta^{\prime}$, including the characterizations of the syntactic consequence statements that involve the negated formula $\neg \varphi$ (cf. items 2 and 4 of Theorem 1).

Theorem 1 thus provides a successful characterization of the syntactic consequence statements in terms of the categorical statements. Hence, just like the square for semantic consequence in Figure 2, the square for syntactic consequence in Figure 3 also closely corresponds to the wellknown square for the categorical statements in Figure 1. However, the former correspondence is directly based on the definition of semantic consequence (cf. Section 2), whereas the latter correspondence is not based on the definition of syntactic consequence (cf. Section 3), but rather on the alternative characterization provided by Theorem 1 (cf. this section). ${ }^{9}$

Nevertheless, this alternative characterization of syntactic consequence can reasonably be understood as the closest possible analogue of the definition of semantic consequence. To see this, recall that the definition of semantic consequence involves categorical statements with subject term 'being a model $M \in \mathcal{C}_{\mathrm{S}}$ such that $M \vDash \Gamma$ ' and predicate term 'being a model $M \in \mathcal{C}_{\mathrm{S}}$ such that $M \vDash \varphi$ ', which are based on the core semantic notion of truth (in a model). The theorem's alternative characterization of syntactic consequence involves categorical statements with subject term 'being an $\operatorname{MCS} \Delta \subseteq \mathcal{L}_{\mathrm{S}}$ such that $\Gamma \subseteq \Delta$ ' and predicate term 'being an MCS $\Delta \subseteq \mathcal{L}_{\mathrm{S}}$ such that $\varphi \in \Delta^{\prime}$, which are based on the corresponding syntactic notion of membership (in a maximal consistent set). More specifically, there exists a direct

\footnotetext{
${ }^{9}$ Furthermore, Theorem 1 cannot be used to provide a definition of syntactic consequence, since that would clearly result in circularity: the theorem characterizes syntactic consequence in terms of MCS, and thus of consistency, but as we have seen in the beginning of Section 3, the latter notion is itself defined in terms of syntactic consequence.
} 
correspondence between maximal consistent sets (that extend $\Gamma$ ) and models (that make all formulas in $\Gamma$ true): one can easily show that (i) if $M$ is a model, then $\Delta_{M}:=\left\{\varphi \in \mathcal{L}_{\mathrm{S}}: M \vDash \varphi\right\}$ is an MCS, and furthermore, (ii) if $M \vDash \Gamma$, then $\Gamma \subseteq \Delta_{M}$. (Note that Henkin-style completeness proofs are based exactly on the fact that $\Gamma \vdash \varphi$ iff for all $\operatorname{MCS} \Delta \supseteq \Gamma$ it holds that $\varphi \in \Delta$.) Furthermore, the close correspondence between membership (in a maximal consistent set $\Delta$ ) and truth (in a model $M$ ) can also be observed in the behavior of negation with respect to both notions: we have $\neg \varphi \in \Delta$ iff $\varphi \notin \Delta$, which is the direct analogue of $M \vDash \neg \varphi$ iff $M \not \varphi$.

Now that we have established a sound analogy between syntactic consequence and the categorical statements, it can again be fruitfully used to understand a certain phenomenon on one side of the analogy in terms of another phenomenon on the other side of the analogy. Consider, for example, the fact that even though $\Gamma \vdash \varphi \vee \neg \varphi$ for all $\Gamma$ and $\varphi$, it is not always the case that $\Gamma \vdash \varphi$ or $\Gamma \vdash \neg \varphi$, which is notoriously difficult for novice logicians to grasp. ${ }^{10}$ However, given the analogy between these two metalogical statements and the categorical Aand E-statements, this fact can straightforwardly be explained. After all, since the A- and Estatements, and thus also the two corresponding metalogical statements, are contraries, they cannot be true together, but they can be false together. The latter means exactly that it can happen that neither $\Gamma \vdash \varphi$ nor $\Gamma \vdash \neg \varphi$. This kind of explanation can be very helpful, for example for philosophers who are thoroughly familiar with syllogistics and the Aristotelian relations, but not with metalogic. Again, note that these observations are perfectly in line with the methodological/heuristic perspective on Aristotelian diagrams.

To finish this section, I would like to emphasize that the analogy between syntactic consequence and the categorical statements described above is by no means unique. Theorem 1 provides an interpretation of the syntactic consequence statements in terms of the categorical statements, which does not suffer from a quantifier mismatch or problems regarding negation. However, one can define further correspondences, which do not suffer from those problems either. For example, instead of working with maximal consistent sets that extend $\Gamma$ (as in Theorem 1), one can also work with all consistent sets that extend $\Gamma$. This idea is made fully precise in Theorem 2 below. Despite the existence of such further interpretations of the syntactic consequence statements in terms of the categorical statements, I do believe that the interpretation described above (in Theorem 1) is the most 'natural' one, because of the aforementioned correspondence

\footnotetext{
${ }^{10}$ The sets $\Gamma$ for which it does hold that $\Gamma \vdash \varphi$ or $\Gamma \vdash \neg \varphi$ for all $\varphi$, are called deductively complete.
} 
between (membership in) a maximal consistent set (that extends $\Gamma$ ) on the one hand, and (truth in) a model (that makes all formulas of $\Gamma$ true) on the other hand.

Theorem 2. Given any set $\Gamma$ of $\mathcal{L}_{\mathrm{S}}$-formulas, and $\mathcal{L}_{\mathrm{S}}$-formula $\varphi$, it holds that:
1) $\Gamma \vdash \varphi$
iff
all consistent $\Gamma^{\prime} \supseteq \Gamma$ are consistent with $\varphi$;
2) $\Gamma \vdash \neg \varphi$
iff no consistent $\Gamma^{\prime} \supseteq \Gamma$ are consistent with $\varphi$;
3) $\Gamma \Vdash \varphi$ iff some consistent $\Gamma^{\prime} \supseteq \Gamma$ are not consistent with $\varphi$;
4) $\Gamma \Vdash \neg \varphi$ iff some consistent $\Gamma^{\prime} \supseteq \Gamma$ are consistent with $\varphi$;
5) $\Gamma$ is consistent iff there exists at least one consistent $\Gamma^{\prime} \supseteq \Gamma$.

Proof. Item 1, left to right. Consider an arbitrary consistent $\Gamma^{\prime} \supseteq \Gamma$ and suppose, toward a contradiction, that $\Gamma^{\prime}$ is not consistent with $\varphi$. Hence $\Gamma^{\prime} \vdash \neg \varphi$. Furthermore, since $\Gamma \vdash \varphi$ and $\Gamma \subseteq \Gamma^{\prime}$, we also have $\Gamma^{\prime} \vdash \varphi$. This violates the consistency of $\Gamma^{\prime}$.

Item 1, right to left is proved by contraposition, so we assume that $\Gamma \nvdash \varphi$, and show that there exists a consistent $\Gamma^{\prime} \supseteq \Gamma$ that is not consistent with $\varphi$. Consider $\Gamma^{\prime}:=\Gamma \cup\{\neg \varphi\}$. It trivially holds that $\Gamma^{\prime} \supseteq \Gamma$; furthermore, $\Gamma^{\prime}$ is consistent because $\Gamma \nvdash \varphi$; finally, $\Gamma^{\prime}$ is not consistent with $\varphi$, since the set $\Gamma^{\prime} \cup\{\varphi\}=\Gamma \cup\{\neg \varphi\} \cup\{\varphi\}$ is trivially not consistent.

Item 2, left to right. Consider an arbitrary consistent $\Gamma^{\prime} \supseteq \Gamma$; we show that $\Gamma^{\prime}$ is not consistent with $\varphi$. Since $\Gamma \vdash \neg \varphi$ and $\Gamma \subseteq \Gamma^{\prime} \subseteq \Gamma^{\prime} \cup\{\varphi\}$, it follows that $\Gamma^{\prime} \cup\{\varphi\} \vdash \neg \varphi$. But trivially also $\Gamma^{\prime} \cup\{\varphi\} \vdash \varphi$, and hence $\Gamma^{\prime} \cup\{\varphi\}$ is not consistent, i.e. $\Gamma^{\prime}$ is not consistent with $\varphi$.

Item 2, right to left is again proved by contraposition, so we assume $\Gamma \Vdash \neg \varphi$, and show that there exists a consistent $\Gamma^{\prime} \supseteq \Gamma$ that is consistent with $\varphi$, viz. $\Gamma$ itself. After all, since $\Gamma \nvdash \neg \varphi$ it follows that $\Gamma$ is consistent with $\varphi$, and thus, a fortiori, that $\Gamma$ itself is consistent.

Items 3 and 4 again follow from items 1 and 2, respectively.

Item 5, left to right. If $\Gamma$ is consistent, there trivially exists at least one consistent $\Gamma^{\prime} \supseteq \Gamma$, viz. $\Gamma$ itself. 
Item 5, right to left is proved by contraposition, so we assume that $\Gamma$ is not consistent, and show that there does not exist a consistent $\Gamma^{\prime} \supseteq \Gamma$. Since $\Gamma$ is not consistent, there exists a formula $\gamma$ such that $\Gamma \vdash \gamma$ and $\Gamma \vdash \neg \gamma$; hence, for every $\Gamma^{\prime} \supseteq \Gamma$ it follows that also $\Gamma^{\prime} \vdash \gamma$ and $\Gamma^{\prime} \vdash \neg \gamma$, and thus $\Gamma^{\prime}$ is not consistent either.

\section{The Role of Soundness and Completeness}

In the previous sections I have focused exclusively on squares of opposition for semantic and syntactic consequence. However, in the literature we also find many larger, more complex metalogical Aristotelian diagrams (see especially Demey and Smessaert 2016 for a comprehensive overview). For example, Béziau $(2012,2013)$ and Diaconescu (2015) show that the square of opposition for semantic consequence (cf. Figure 2) can be extended to a so-called Jacoby-Sesmat-Blanché (JSB) hexagon of opposition, by adding two further semantic consequence statements, viz. the disjunction of $\Gamma \vDash \varphi$ and $\Gamma \vDash \neg \varphi$ (the syntactic counterpart of which is used in the definition of deductively complete sets; cf. Footnote 10), and the conjunction of $\Gamma \not \models \varphi$ and $\Gamma \not \neg \varphi$. It is straightforward to show that the square of opposition for syntactic consequence (cf. Figure 3) can also be extended to a JSB hexagon, in a completely analogous fashion. However, in this section I want to focus on another type of metalogical Aristotelian diagrams, in which the semantic and syntactic consequence statements appear simultaneously, and which are thus also able to capture the interaction between these two types of statements. The specific details of these diagrams depend on whether we assume the logical system $\mathrm{S}$ to be sound and/or complete - or more precisely: whether we assume the proof system $\mathcal{D}_{\mathrm{S}}$ to be sound and/or complete with respect to the class of models $\mathcal{C}_{\mathrm{S}}$. I will now systematically discuss each of the four possible combinations of soundness/completeness assumptions, and the Aristotelian diagram that they give rise to. ${ }^{11}$

\footnotetext{
${ }^{11}$ Many logicians have a rather 'asymmetric' perspective on soundness and completeness: soundness is usually taken to be a kind of minimal criterion that has to be met by any serious candidate proof system, while failure of completeness is seen as much more tolerable. However, based on certain technical results on so-called Scott consequence relations, one can argue for a more 'symmetric' perspective, which treats soundness and completeness more on a par with each other (Scott 1971, 1974, Brown 2015). In particular, a logical system that is complete but not sound is deemed equally worthy of attention as a system that is sound but not complete. Thanks to an anonymous reviewer for some interesting remarks about this issue.
} 
First of all, let's start by assuming neither that the logical system $S$ is sound, nor that it is complete. In this situation, putting the four semantic consequence statements and the four syntactic consequence statements together does not yield an interesting new Aristotelian diagram. After all, we will have a square of opposition for semantic consequence (under the assumption of satisfiability of $\Gamma$; cf. Section 2) and a square of opposition for syntactic consequence (under the assumption of consistency of $\Gamma$; cf. Section 3), but because we assume neither soundness nor completeness, these two squares will not enter into any interaction with each other. In particular, there will not be any Aristotelian relation holding between a semantic consequence statement on the one hand, and a syntactic consequence statement on the other.

Secondly, let's assume that the logical system S is sound, but not necessarily complete. Based on this assumption (along with the assumptions regarding the satisfiability and consistency of $\Gamma$ from Sections 2 and 3), one can construct an octagon of opposition for semantic and syntactic consequence as shown in Figure 4. This type of Aristotelian diagram is sometimes called a 'Lenzen octagon', since (a visual variant of) it was first studied by Lenzen (2012).

\section{$<$ INCLUDE FIGURE 4 HERE $>$}

$<$ CAPTION: Figure 4: metalogical octagon of opposition for semantic and syntactic consequence, under the assumptions that $\Gamma$ is satisfiable and consistent, and that $S$ is sound.>

This octagon incorporates (a horizontally stretched version of) the square for semantic consequence, as well as (a vertically stretched version of) the square for syntactic consequence as subdiagrams. Furthermore, it also shows the Aristotelian relations capturing the interaction between these two squares. For example, there is a subalternation from $\Gamma \vdash \varphi$ to $\Gamma \vDash \varphi$ : because of soundness, $\Gamma \vdash \varphi$ indeed entails $\Gamma \vDash \varphi$, but since the proof system is not assumed to be complete, the other direction does not generally hold. The same considerations also show that there is a subalternation from $\Gamma \not \varphi$ to $\Gamma \Vdash \varphi$, that $\Gamma \vdash \varphi$ is contrary to $\Gamma \not \varphi$, and that $\Gamma \vDash \varphi$ is subcontrary to $\Gamma \Vdash \varphi$. Furthermore, by composing the subalternation from $\Gamma \vdash \varphi$ to $\Gamma \vDash \varphi$ with the subalternation from $\Gamma \vDash \varphi$ to $\Gamma \not \neg \varphi$ in the semantic square, we also obtain the subalternation from $\Gamma \vdash \varphi$ to $\Gamma \not \neg \varphi$. All the Aristotelian relations shown in Figure 4 can straightforwardly be established in this manner. 
The Lenzen octagon in Figure 4 can thus be partitioned into three parts, each of which depends on its own assumption: (i) the square of opposition for semantic consequence, which depends on the satisfiability of $\Gamma$, (ii) the square of opposition for syntactic consequence, which depends on the consistency of $\Gamma$, and (iii) the Aristotelian relations capturing the interaction between these first two squares, which depend on the soundness of S. (Note that these interaction relations can themselves be clustered into four additional squares of opposition.) This Aristotelian diagram is thus a compact yet systematic visualization of the 8 metalogical statements about semantic and syntactic consequence, and the logical relations holding between them under the assumption of soundness. Such a diagram can be very helpful for those who are new to metalogic in general, and to notions such as satisfiability, consistency and soundness in particular.

Thirdly, let's now switch to the 'symmetric' situation, and assume that the logical system $\mathrm{S}$ is complete, but not necessarily sound (recall Footnote 11). Based on this assumption (along with the assumptions regarding the satisfiability and consistency of $\Gamma$ from Sections 2 and 3), one can again construct a Lenzen octagon for semantic and syntactic consequence, as shown in Figure 5 .

\section{$<$ INCLUDE FIGURE 5 HERE $>$}

$<$ CAPTION: Figure 5: metalogical octagon of opposition for semantic and syntactic consequence, under the assumptions that $\Gamma$ is satisfiable and consistent, and that $S$ is complete. $>$

This Lenzen octagon can again be partitioned into three parts, each of which depends on its own assumption: (i) the square of opposition for semantic consequence (now vertically stretched), which depends on the satisfiability of $\Gamma$, (ii) the square of opposition for syntactic consequence (now horizontally stretched), which depends on the consistency of $\Gamma$, and (iii) the Aristotelian relations capturing the interaction between these first two squares, which depend on the completeness of S. (Again, note that these interaction relations can themselves be clustered into four additional squares of opposition.)

Fourthly, let's examine what happens if we assume that $\mathrm{S}$ is sound and complete (while still maintaining the assumptions regarding the satisfiability and consistency of $\Gamma$ ). Obviously, this joint assumption will not have any impact on the squares of opposition for semantic and 
syntactic consequence separately, but only on their interaction. However, it influences the Aristotelian relations capturing this interaction in a highly heterogeneous fashion.

On the one hand, consider the effects on the first Lenzen octagon, which only assumed soundness (cf. Figure 4). The subalternation from $\Gamma \vdash \varphi$ to $\Gamma \vDash \varphi$ in that octagon will turn into an equivalence: because we now assume soundness and completeness, these two statements entail each other. (The same consideration also shows that the octagon's contrariety between $\Gamma \vdash \varphi$ and $\Gamma \not \varphi$ now turns into a contradiction.) By contrast, the octagon's subalternation from $\Gamma \vdash \varphi$ to $\Gamma \not \neg \varphi$ remains a subalternation: even under the additional assumption of completeness, $\Gamma \not \neg \neg \varphi$ still does not entail $\Gamma \vdash \varphi$. (The same consideration also shows that the octagon's contrariety between $\Gamma \vdash \varphi$ and $\Gamma \vDash \neg \varphi$ remains a contrariety.)

On the other hand, consider the effects on the second Lenzen octagon, which only assumed completeness (cf. Figure 5). The subalternation from $\Gamma \vDash \varphi$ to $\Gamma \vdash \varphi$ in this second octagon will turn into an equivalence: because we now assume completeness and soundness, these two statements entail each other. (The same consideration also shows that the octagon's contrariety between $\Gamma \vDash \varphi$ and $\Gamma \Vdash \varphi$ now turns into a contradiction.) By contrast, the octagon's subalternation from $\Gamma \vDash \varphi$ to $\Gamma \Vdash \neg \varphi$ remains a subalternation: even under the additional assumption of soundness, $\Gamma \nvdash \neg \varphi$ still does not entail $\Gamma \vDash \varphi$. (The same consideration also shows that the octagon's contrariety between $\Gamma \vDash \varphi$ and $\Gamma \vdash \neg \varphi$ remains a contrariety.)

\section{$<$ INCLUDE FIGURE 6 HERE $>$}

$<$ CAPTION: Figure 6: metalogical octagon of opposition for semantic and syntactic consequence, under the assumptions that $\Gamma$ is satisfiable and consistent, and that $S$ is sound and complete.>

All these changes can be summarized as follows: under the joint assumption of soundness and completeness, the Lenzen octagons from Figures 4 and 5 both turn into a new octagon of opposition, as shown in Figure 6. (Note that bidirectional arrows are used to visualize mutual entailment, i.e. equivalence, between statements.) Once again, this octagon can be partitioned into three parts, each of which depends on its own assumption: (i) the (horizontally stretched) square of opposition for semantic consequence, which depends on the satisfiability of $\Gamma$, (ii) the (vertically stretched) square of opposition for syntactic consequence, which depends on the 
consistency of $\Gamma$, and (iii) the mutual entailments and Aristotelian relations capturing the interaction between these first two squares, which depend on the soundness and completeness of S.

The octagon of opposition in Figure 6 visualizes each of the eight metalogical statements by means of a separate vertex in the diagram, despite these statements being pairwise equivalent to each other (under the joint assumption of soundness and completeness). This visualization strategy is not entirely in line with common practice in philosophical logic: most Aristotelian diagrams that appear in the literature do not contain equivalent statements at all (cf. Figures 1 -5 in this paper as well), but among those diagrams that $d o$ contain equivalent statements, the overwhelming majority visualizes equivalent statements by assigning them to the same vertex. ${ }^{12}$ If we apply this more canonical visualization strategy to the octagon in Figure 6, then this octagon 'collapses' into a square of opposition, as shown in Figure 7. Each vertex of this square is occupied by two equivalent metalogical statements, and each Aristotelian relation between two vertices holds between either formula in one vertex and either formula in the other. Essentially, the square of opposition in Figure 7 can be seen as the result of superimposing the squares for semantic consequence (Figure 2) and for syntactic consequence (Figure 3). Both the octagon in Figure 6 and its 'collapsed' square version in Figure 7 can again be very helpful for those who are new to metalogic in general, and to notions such as soundness and completeness in particular.

\section{$<$ INCLUDE FIGURE 7 HERE $>$}

$<$ CAPTION: Figure 7: metalogical square of opposition for semantic and syntactic consequence, under the assumptions that $\Gamma$ is satisfiable and consistent, and that $S$ is sound and complete.>

In Sections 2 and 3, I already pointed out that Aristotelian diagrams are sensitive with respect to certain background assumptions: adding or dropping the assumption that $\Gamma$ is satisfiable/consistent makes the Aristotelian diagrams for semantic/syntactic consequence switch between two types of squares (viz. a classical square of opposition vs. a degenerate square). In this section, however, we have encountered a much more radical manifestation of

\footnotetext{
${ }^{12}$ Cf. Lenzen (2017, p. 11) for a typical example, and Smessaert and Demey (2014, pp. 530-531) for further discussion.
} 
this general phenomenon. For example, if we keep the assumption of soundness fixed, then adding or dropping the assumption of completeness makes the Aristotelian diagram for the interaction between semantic and syntactic consequence switch between two very different types of diagrams (viz. the Lenzen octagon in Figure 4 vs. the classical square of opposition in Figure 7). And vice versa, if we keep the assumption of completeness fixed, then adding or dropping the assumption of soundness makes the Aristotelian diagram for the interaction between semantic and syntactic consequence again switch between a Lenzen octagon (Figure 5) and a classical square of opposition (Figure 7).

\section{Conclusion}

In this paper I have shown that syntactic logical consequence gives rise to a metalogical square of opposition, just like semantic logical consequence. Unlike the definitions of the semantic consequence statements, the definitions of the syntactic consequence statements cannot be interpreted in terms of the categorical statements from Aristotelian syllogistics. Nevertheless, the theorem proved in Section 4 provides an alternative characterization of syntactic consequence, thereby yielding another, equally elegant interpretation in terms of the categorical statements. Hence, we now have a semantic consequence square as well as a syntactic consequence square, both of which exhibit a strong and potentially very useful analogy with the categorical statements from syllogistics. I have also constructed a metalogical Aristotelian diagram that incorporates both semantic and syntactic consequence, as well as the interaction between them (as determined by the soundness and/or completeness of the underlying logical system). Along the way, I have pointed out several metalogical manifestations of a broader phenomenon that is well-known in logical geometry, viz. the idea that Aristotelian diagrams can be sensitive with respect to certain background assumptions. We encountered relatively mild cases of this phenomenon (classical square of opposition vs. degenerate square), but also more radical cases (Lenzen octagon vs. classical square of opposition).

All of this provides further support for the methodological/heuristic perspective on Aristotelian diagrams, which holds that the main use of these diagrams lies in facilitating analogies and comparisons between prima facie unrelated domains of investigation. Most specific examples that have hitherto been adduced in favor of this perspective, concern analogies between systems that are both situated at the object-logical level, such as Russell's theory of definite descriptions 
and public announcement logic. By contrast, the cases discussed in this paper cut across the object-/meta-level divide, and are concerned with analogies between the metalogical notions of semantic and syntactic consequence and the object-logical categorical statements. This shows that the methodological/heuristic potential of Aristotelian diagrams might be even larger than has previously been thought.

Throughout the paper, I have already briefly hinted at the methodological potential of the new metalogical diagrams that have been developed here (cf. Figures $3-7$ ), for example regarding the entailment from $\Gamma \vDash \varphi$ to $\Gamma \not \models \neg \varphi$ and its dependence on the assumption that $\Gamma$ be satisfiable (cf. Section 2), and the fact that it is not always the case that $\Gamma \vdash \varphi$ or $\Gamma \vdash \neg \varphi$ (cf. Section 4 ). In future work I will explore this methodological potential in a more systematic fashion. The elegant yet simple characterization of syntactic consequence in terms of the categorical statements (cf. the theorems proved in Section 4) will likely be of crucial importance in this respect.

\section{Acknowledgements}

Thanks to Hans Smessaert, Margaux Smets and three anonymous reviewers for their valuable feedback on an earlier version of this paper. The author holds a Postdoctoral Fellowship of the Research Foundation-Flanders (FWO). The research reported in this paper was partially carried out during a research stay at the Institut für Philosophie II of the Ruhr-Universität Bochum, which was financially supported by an FWO travel grant.

\section{Bibliography}

R. Barcan Marcus. Iterated Deontic Modalities. Mind 75 (1966), 580 - 582.

J. C. Beall, R. T. Brady, A. P. Hazen, G. Priest, and G. Restall. Relevant Restricted Quantification. Journal of Philosophical Logic 35 (2006), 587 - 598.

J.-Y. Béziau. The Power of the Hexagon. Logica Universalis 6 (2012), 1 - 43. 
J.-Y. Béziau. The Metalogical Hexagon of Opposition. Argumentos 5 (2013), 111 - 122.

J.-Y. Béziau. Disentangling Contradiction from Contrariety via Incompatibility. Logica Universalis 10 (2016), $157-170$.

J.-Y. Béziau and G. Payette. Preface. In: J.-Y. Béziau and G. Payette (eds.), The Square of Opposition. A General Framework for Cognition, Peter Lang, 2012, p. 9 - 22.

B. Brown. Stipulation and Symmetrical Consequence. In: J.-Y. Béziau, M. Chakraborty and S. Dutta (eds.), New Directions in Paraconsistent Logic, Springer, 2015, p. 335 - 352.

R. Chisholm. Supererogation and Offence: A Conceptual Scheme for Ethics. Ratio 5 (1963), 1 -14 .

D. Ciucci, D. Dubois and H. Prade. The Structure of Opposition in Rough Set Theory and Formal Concept Analysis. Toward a Bridge between the Two Settings. In: C. Beierle and C. Meghini (eds.), Foundations of Information and Knowledge Systems (FoIKS 2014), Springer, 2014, p. $154-173$.

D. Ciucci, D. Dubois, and H. Prade. Structures of Opposition Induced by Relations. The Boolean and the Gradual Cases. Annals of Mathematics and Artificial Intelligence 76 (2016), $351-373$.

L. Demey. Structures of Oppositions in Public Announcement Logic. In: J.-Y. Béziau and D. Jacquette (eds.), Around and Beyond the Square of Opposition, Springer, 2012, p. 313 - 339.

L. Demey. Interactively Illustrating the Context-Sensitivity of Aristotelian Diagrams. In: H. Christiansen, I. Stojanovic, and G. Papadopoulous (eds.), Modeling and Using Context, Lecture Notes in Computer Science 9405, Springer, 2015, p. $331-345$.

L. Demey. Using Syllogistics to Teach Metalogic. Metaphilosophy 48 (2017a), 575 - 590.

L. Demey. The Logical Geometry of Russell's Theory of Definite Descriptions. Unpublished manuscript (2017b). 
L. Demey. Aristotelian Diagrams in the Debate on Future Contingents. Sophia (2018a).

L. Demey. Metalogic, Metalanguage, and Logical Geometry. Logique et Analyse (2018b).

L. Demey. The Role of Aristotelian Diagrams in Scientific Communication. Talk delivered at the $8^{\text {th }}$ Visual Learning Conference, Budapest (2018c).

L. Demey and H. Smessaert. Logische geometrie en pragmatiek. In: F. Van de Velde, H. Smessaert, F. Van Eynde, and S. Verbrugge (eds.), Patroon en argument, Leuven University Press, 2014, p. $553-564$.

L. Demey and H. Smessaert. Metalogical Decorations of Logical Diagrams. Logica Universalis 10 (2016), $233-292$.

L. Demey and H. Smessaert. Combinatorial Bitstring Semantics for Arbitrary Logical Fragments. Journal of Philosophical Logic 47 (2018), 325 - 363.

R. Diaconescu. The Algebra of Opposition (and Universal Logic Interpretations). In: A. Koslow and A. Buchsbaum (eds.), The Road to Universal Logic, Springer, 2015, p. 127 - 143.

D. Dubois, H. Prade and A. Rico. The Cube of Opposition. A Structure underlying many Knowledge Representation Formalisms. In: Q. Yang and M. Wooldridge (eds.), Proceedings of the Twenty-Fourth International Joint Conference on Artificial Intelligence (IJCAI 2015), AAAI Press, 2015, p. 2933 - 2939.

R. M. Hare. Some Alleged Differences between Imperatives and Indicatives. Mind 76 (1967), $309-326$.

H. L. A. Hart. Essays on Bentham. Jurisprudence and Political Theory. Clarendon Press, 1982.

A. Kenny. Action, Emotion and Will. Routledge \& Kegan Paul, 1963. 
W. Kienzler. The Logical Square and the Table of Oppositions. Five Puzzles about the Traditional Square of Opposition Solved by Taking Up a Hint from Frege. Logical Analysis and History of Philosophy 15 (2013), 398 - 413.

W. Lenzen. How to Square Knowledge and Belief. In: J.-Y. Béziau and D. Jacquette (eds.), Around and Beyond the Square of Opposition, Springer, 2012, p. 305 - 311.

W. Lenzen. Leibniz's Logic and the "Cube of Opposition”. Logica Universalis 10 (2016), 171 $-189$.

W. Lenzen. Caramuel's Theory of Opposition. South American Journal of Logic 3 (2017), 1 27.

T. Parsons. The Traditional Square of Opposition. In: E. N. Zalta (ed.), Stanford Encyclopedia of Philosophy (Summer 2017 Edition), 2017.

N. Pfeifer and G. Sanfilippo. Probabilistic Squares and Hexagons of Opposition under Coherence. International Journal of Approximate Reasoning 88 (2017), 282 - 294.

A. Rini and M. Cresswell. The World-Time Parallel. Tense and Modality in Logic and Metaphysics. Cambridge University Press, 2012.

D. Scott. On Engendering an Illusion of Understanding. Journal of Philosophy 68 (1971), 787 $-807$.

D. Scott. Completeness and Axiomatizability in Many-Valued Logic. In: L. Henkin (ed.), Proceedings of the Tarski Symposium, American Mathematical Society, 1974, p. 411 - 435.

P. Seuren. Metalogical Hexagons in Natural Logic. Unpublished manuscript (2014).

E. Sosa. The Analysis of 'Knowledge that P'. Analysis 25 (1964), 1 - 8.

H. Smessaert and L. Demey. Logical Geometries and Information in the Square of Oppositions. Journal of Logic, Language and Information 23 (2014), 527 - 565. 
E. Vranes. The Definition of 'Norm Conflict' in International Law and Legal Theory. European Journal of International Law 17 (2006), 395 - 418.

Y. Yao. Duality in Rough Set Theory based on the Square of Opposition. Fundamenta Informaticae 127 (2013), 49 - 64.

D. Ziegeler. On the Empty O-Corner of the Aristotelian Square: A View from Singapore English. Journal of Pragmatics 115 (2017), 1 - 20. 
$<$ FIGURE 1>
A: All S are $\mathrm{P}$
E: No $\mathrm{S}$ are $\mathrm{P}$

contradiction

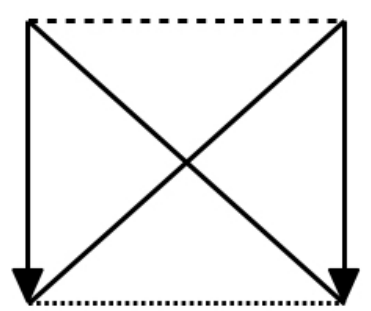

subalternation

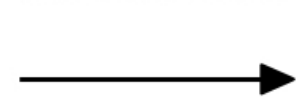

I: Some $\mathrm{S}$ are $\mathrm{P}$

$\mathrm{O}$ : Some $\mathrm{S}$ are not $\mathrm{P}$

$<$ FIGURE 2>
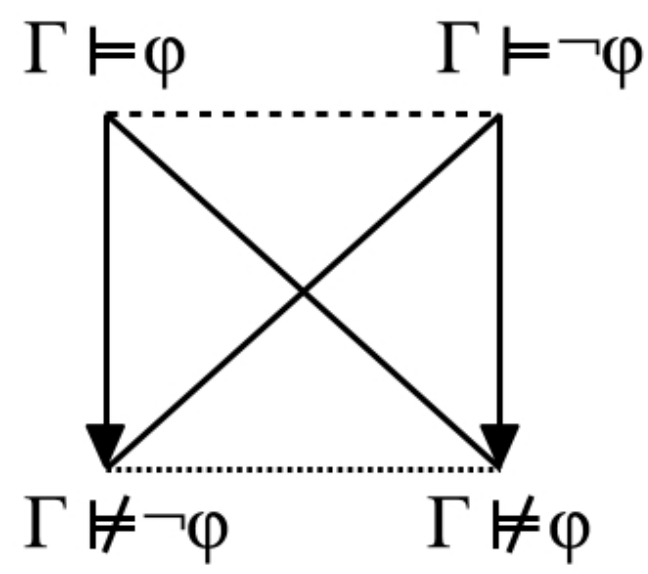

$<$ FIGURE 3>

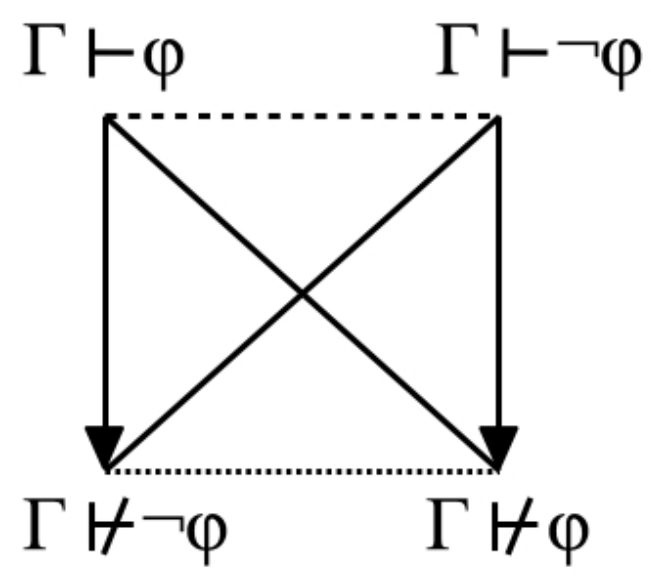


$<$ FIGURE 4>

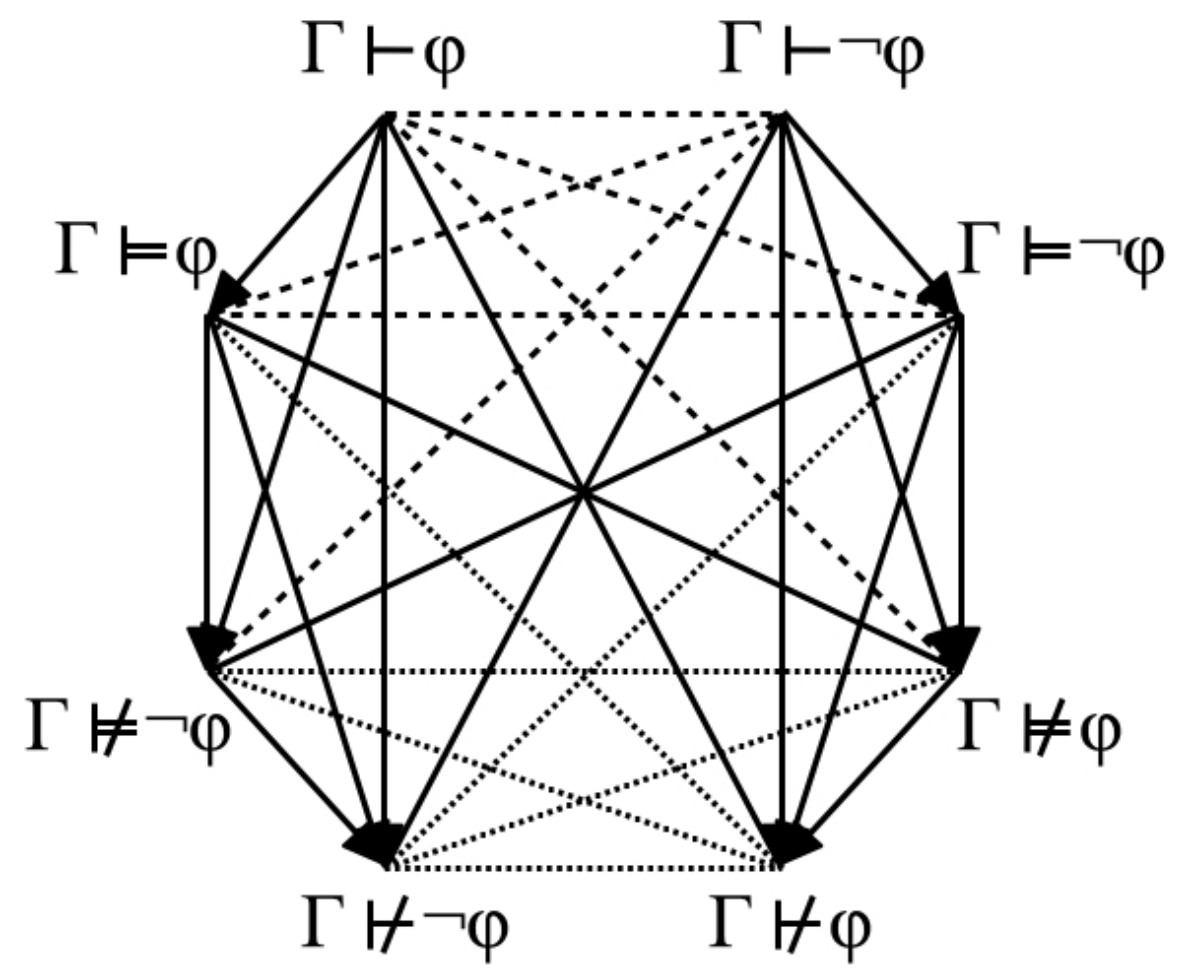

$<$ FIGURE 5>

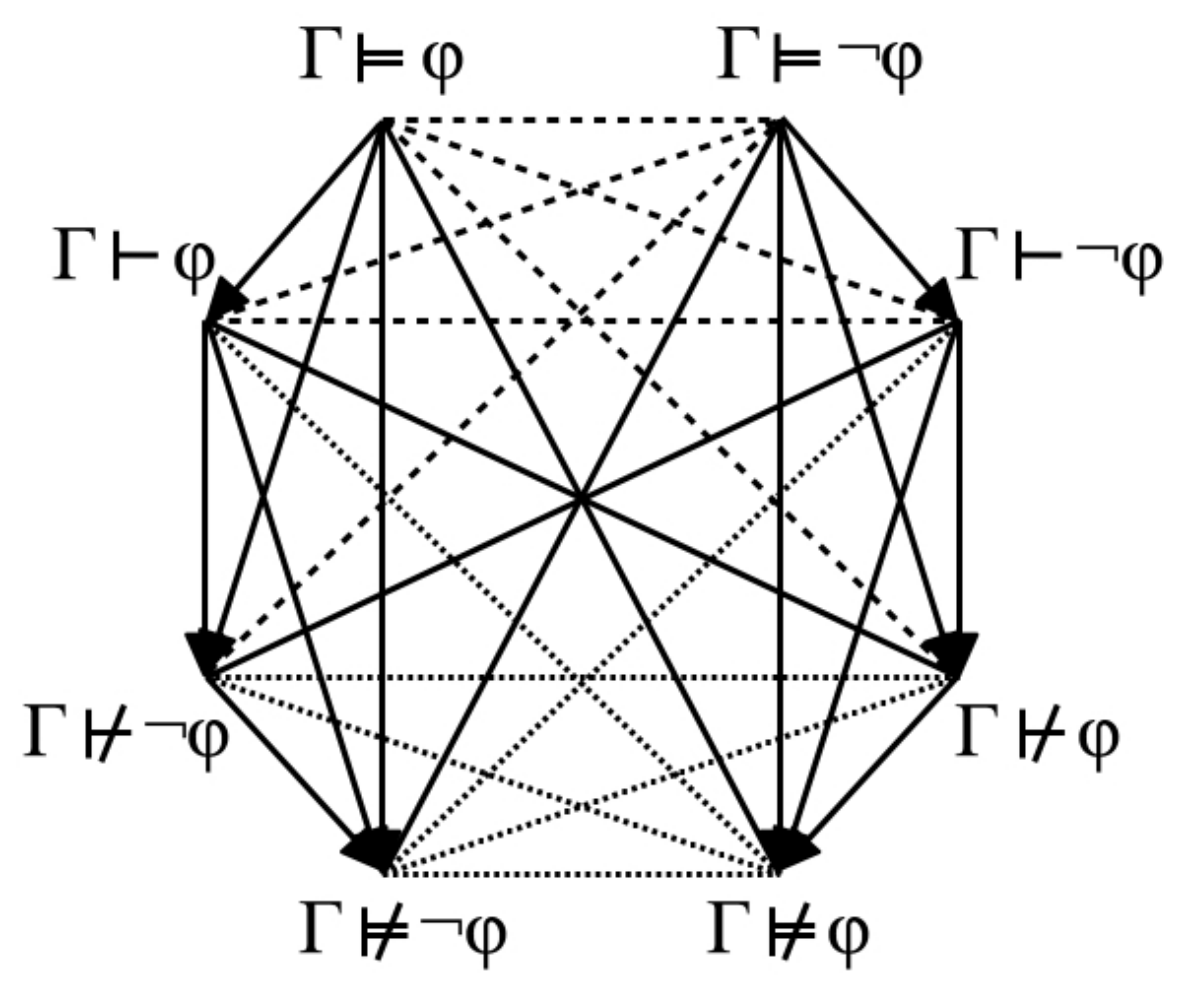


$<$ FIGURE 6>

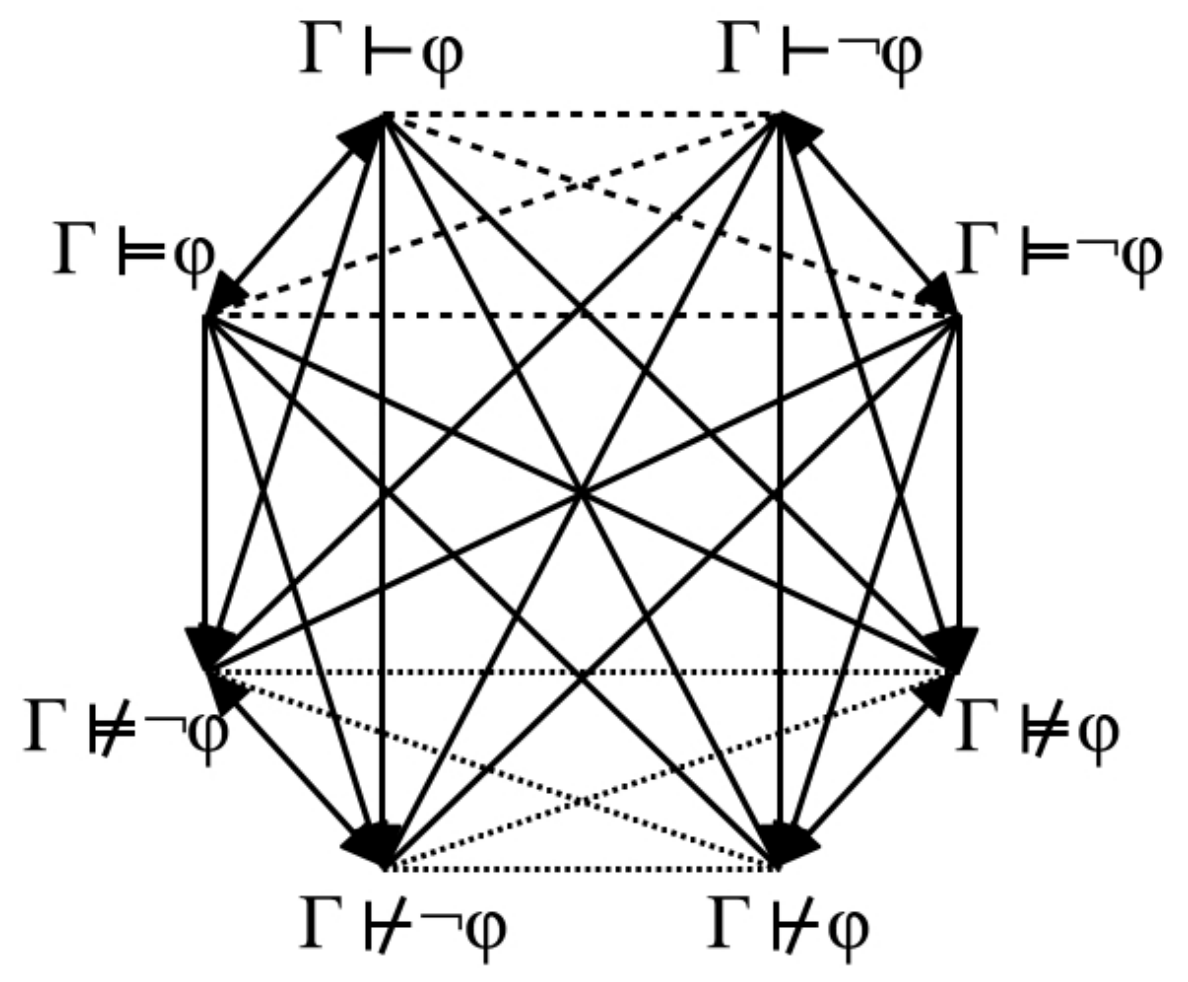

$<$ FIGURE 7>

$\begin{array}{ll}\Gamma \models \varphi & \Gamma \vDash \neg \varphi \\ \Gamma \vdash \varphi & \Gamma \vdash \neg \varphi\end{array}$

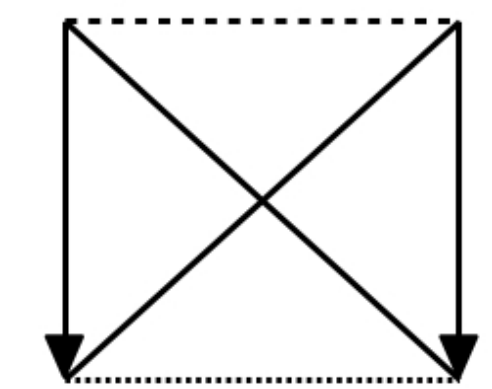

$\Gamma \nvdash \neg \varphi \quad \Gamma \nvdash \varphi$

$\Gamma \not \neg \varphi \quad \Gamma \not \varphi$ 